\section{SNARE derived peptide mimic inducing membrane fusion $\dagger$}

\author{
Karsten Meyenberg, ${ }^{a}$ Antonina S. Lygina, ${ }^{a}$ Geert van den Bogaart, ${ }^{b}$ Reinhard Jahn ${ }^{b}$ and \\ Ulf Diederichsen $* a$
}

Received 16th May 2011, Accepted 28th June 2011
DOI: 10.1039/c1cc12879e

SNARE proteins mediate membrane fusion between synaptic vesicles and the plasma membrane. A minimized peptide SNARE model system with reduced complexity was introduced combining the native SNARE transmembrane (TMD) and linker domains with artificial coiled-coil forming peptides. Specific membrane fusion initiated by coiled-coil recognition was shown by lipid and content mixing vesicle assays.

Membrane fusion is a central cellular process in eukaryotic cells. In the secretory pathway connecting organelles between the endoplasmic reticulum and the plasma membrane, fusion is mediated by sets of SNARE proteins (soluble $\mathbf{N}$-ethylmaleimide-sensitive factor attachment receptor). ${ }^{1}$ Complementary sets of SNARE proteins are associated with the respective membranes. Upon contact, the SNAREs form a thermodynamically stable four helix bundle connecting the membranes (trans-complex) probably being generated in a zipper-like recognition process followed by helicalization. ${ }^{2,3}$ Thereby, the membranes are brought into proximity overcoming the energy barrier for membrane fusion. ${ }^{4}$

Neuronal exocytosis is catalyzed by the SNARE proteins SNAP-25 (two $\alpha$-helices), VAMP2 (synaptobrevin), and syntaxin-1A (each one $\alpha$-helix), with the two latter each possessing a single transmembrane domain (TMD) at the $C$-terminus (Fig. 1a). ${ }^{1,5}$ In vitro reconstitution of these SNAREs in liposomes ranging between 30-100 nm diameter results in SNARE-mediated membrane fusion. ${ }^{6}$ However, both assembly and fusion kinetics in these systems are highly complex; and it is difficult to differentiate between parameters affecting nucleation and zippering of the helix bundle and the downstream events of membrane contact, hemifusion, and fusion. In order to derive mechanistic insight into the fusion event, simpler model systems are needed. In particular, substitution of the SNARE complex by SNARE-mimicking peptides may shed light on the influence of the TMDs and the linker regions between the SNARE complex forming helices and the TMDs. In addition, investigation of various artificial recognition motifs

${ }^{a}$ Institut für Organische und Biomolekulare Chemie, Tammannstr. 2, 37077 Göttingen, Germany. E-mail: udieder@gwdg.de;

Fax: 49 551-39 22944; Tel: 49551393221

${ }^{b}$ Max-Planck-Institut für Biophysikalische Chemie, Am Faßberg 11, 37077 Göttingen, Germany

$\dagger$ Electronic supplementary information (ESI) available. See DOI: $10.1039 / \mathrm{clcc} 12879 \mathrm{e}$

opens the chance to investigate the molecular requirements fulfilled by SNARE complex formation.

Several SNARE-mimicking model systems have been reported with the aim of reducing complexity in membrane fusion. ${ }^{7}$ Complementary artificial recognition motifs were linked to cholesterol, ${ }^{8,9}$ phospholipids, ${ }^{10-15}$ or membrane penetrating peptides $^{14,15}$ and were subsequently embedded into the membrane. A variety of chemically diverse recognition motifs for linking the membranes has been reported such as the reversible reaction between boronic acid and diols, ${ }^{13}$ DNA double strands, ${ }^{8,9,11,12}$ and a vancomycin glycopeptide combined with a D-Ala-D-Ala moiety. ${ }^{14,15}$ Another reduced SNARE model has been introduced that is based on a coiled-coil forming $\alpha$-peptide. ${ }^{10}$ Although in all of these cases specific membrane fusion was observed, they are structurally different from the SNAREs, and thus, do not allow for direct mechanistic extrapolation to SNARE-mediated fusion events.

To overcome this limitation, we have recently introduced a new SNARE-mimicking model using the native TMDs linked to peptide nucleic acid (PNA) recognition motifs and obtained efficient fusion that proceeds via hemifusion as intermediate. ${ }^{16}$ We have now extended this approach by employing coiled-coil forming $\alpha$-peptides. ${ }^{10}$ To this end, we have replaced the four SNARE helices (Fig. 1a) by smaller coiled-coil forming $\alpha$-peptides (Fig. 1b and c). The hybrid between an $\alpha$-peptide recognition motif and the SNARE derived TMDs is advantageous since it allows for studying the role of the native linkers and TMDs of synaptobrevin and syntaxin in the fusion reaction. Here, we report about the synthesis of these molecules and

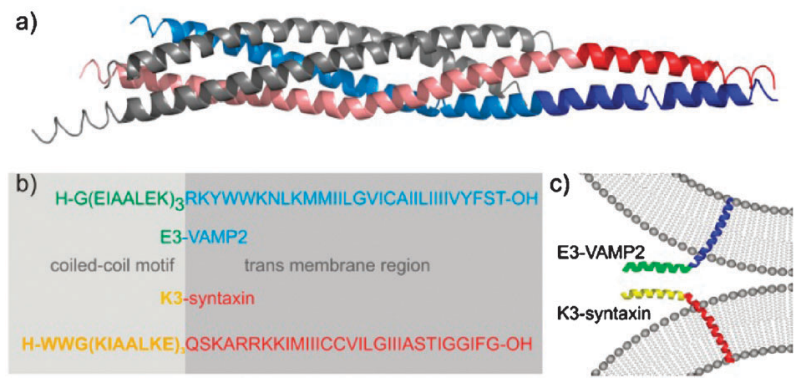

Fig. 1 (a) Crystal structure of the synaptic ternary SNARE cis-complex (syntaxin1A — red, VAMP2 — blue, SNAP25 — grey; rattus norvegicus).$^{17}$ (b) Sequence of the peptide SNARE analogues with sequentially identical TMD domains. (c) Membrane incorporation and proposed interaction of the peptide/TMDs K3-syntaxin and E3-VAMP2 embedded in opposing membranes. 
show that efficient fusion can be achieved by these SNAREmimicking molecules.

The peptide fragments $\mathrm{H}-\mathrm{WWG}(\mathrm{KIAALKE})_{3}-\mathrm{OH}$ (K3) and $\mathrm{H}-\mathrm{G}(\mathrm{EIAALEK})_{3}-\mathrm{OH}$ (E3) were selected as coiled-coil recognition unit. ${ }^{10}$ Both consist of three heptad repeats that assemble by electrostatic and hydrophobic core interactions. They form a stable heterodimer originally designed to serve as biosensor or affinity purification tag. The peptides interact with a $\mathrm{k}_{\mathrm{D}}$ of $10^{-7} \mathrm{~mol} \mathrm{~L}^{-1}$ and require a free energy of $9.6 \mathrm{kcal} \mathrm{mol}^{-1}$ to unfold. ${ }^{18}$ Therefore, the free energy is qualitatively in good agreement with the energy provided by the four helix bundle of the neuronal SNARE complex $\left(\sim 20 \mathrm{kcal} \mathrm{mol}^{-1}\right) .{ }^{19}$ The coiled-coil forming peptides were $\mathrm{N}$-terminally linked to the TMDs of the neuronal SNARE proteins VAMP2 (residues 85-116) and syntaxin-1A (258-288). The SNARE hybrids K3-syntaxin, E3-VAMP2 and respective reference peptides were synthesized by means of automated microwave assisted solid phase peptide synthesis (SPPS) and characterized by ESI mass spectrometry (supporting information $\dagger$ ).

In order to investigate whether the peptides are able to induce membrane fusion, the peptides were reconstituted into liposomes. ${ }^{20}$ Fusion was measured by a FRET assay for lipid mixing (Fig. 2) using the FRET pair 1, 1'dioctadecyl-3,3,3',3'tetramethylindodicarbocyanine perchlorate (DiD, acceptor) and oregon green (OG, donor). ${ }^{6}$ The peptides were incorporated into liposomes in a $1 / 2000$ peptide to lipid molar ratio using size exclusion chromatography. ${ }^{21}$ The membranes were composed of a $5: 2: 2: 1$ molar ratio of phosphatidyl choline, phosphatidyl ethanol amine, phosphatidyl serine (all from pig brain) and cholesterol as this mixture mimics the composition of synaptic membranes. ${ }^{22}$ Liposomes ( $80 \mu \mathrm{M}$ total lipids) labelled with oregon green 488 1,2-dihexadecanoyl-sn-glycero-3-phosphoethanolamine (OG-DHPE, $1.5 \mathrm{~mol} \%$ ) and containing $\mathrm{K} 3$-syntaxin were mixed with DiD (1.5 mol\%) labelled liposomes $(80 \mu \mathrm{M})$ containing E3-VAMP2 in buffer solution at room temperature (Fig. 2).

Lipid mixing of both liposomes was indicated by an increase of the acceptor fluorescence over a period of $20 \mathrm{~min}$ due to energy transfer from the OG to the DiD-fluorophor in the resulting membrane. In order to prove that lipid mixing was initiated by coiled-coil recognition, a competition experiment was performed adding the soluble peptide $\mathrm{H}-\mathrm{G}\left(\right.$ EIAALEK) ${ }_{3} \mathrm{G}-\mathrm{OH}$ $\left(\mathrm{E}^{\prime}\right)(4 \mu \mathrm{M})$ to the liposomes containing the K3-syntaxin

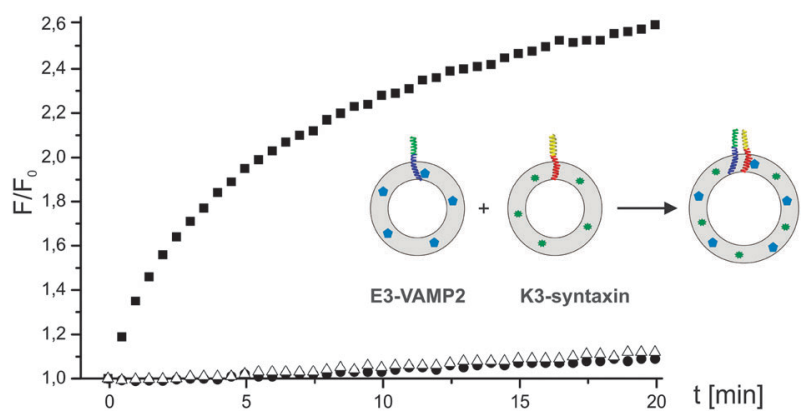

Fig. 2 Lipid mixing of liposomes containing SNARE-mimicking peptides using a fluorescence dequenching assay. E3-VAMP2 and K3-syntaxin ( $\mathbf{\square})$; addition of peptide E3 to K3-syntaxin liposomes prior to fusion with E3-VAMP2 (-); vesicle populations both containing E3-VAMP2 $(\triangle)$. hybrid prior to the fusion experiment. Almost no change in fluorescence was observed in the mixing process with E3-VAMP2 liposomes. Fluorescence anisotropy measurements confirmed the binding of the soluble E3' peptide to the membrane bound K3-syntaxin, thereby, inhibiting recognition of the peptides bound in opposing membranes (supporting information Fig. S1). As a second control for the dependence of fusion on coiled-coil formation we incubated DiD and OG labelled vesicles both containing E3-VAMP2. Almost no change in fluorescence was observed. Then, peptides were synthesized in which the recognition units between the VAMP and syntaxin fragments were swapped (K3-VAMP2 and E3-syntaxin). Again, a robust increase of fluorescence was observed that was comparable to lipid mixing of vesicles mediated by K3-syntaxin and E3-VAMP2 (supporting information Fig. S2 $\dagger$ ). Together, these results show that replacement of the conserved four helix bundle by a simple binary recognition motif that is fused to the linkers and transmembrane domains of the neuronal SNAREs suffices to mediate membrane fusion. Their specific recognition seems to be essential for membrane fusion, regardless to which transmembrane segments the recognition motifs are linked.

To compare the kinetics of membrane fusion of our minimized peptide fusion models with that of native SNAREs, two different and commonly used in vitro set-ups for native SNAREs ${ }^{23}$ were employed. Firstly, syntaxin-1A (lacking its inhibitory Habc domain; residue $183-288)^{24}$ containing vesicles were preincubated with SNAP25 yielding the syntaxin-1A/SNAP25 complex $(2: 1$ complex) ${ }^{25}$ Lipid mixing was measured with vesicles containing this $2: 1$ complex with VAMP2 vesicles (Fig. 3). In this fusion assay, the fluorescence increase was about five times slower than that obtained with the SNARE-mimicking peptides.

Secondly, we replaced the 2:1 complex with a stabilized syntaxin/SNAP25 acceptor complex consisting of syntaxin-1A (183-288), SNAP-25, and a short fragment of VAMP2 (49-96; $\Delta \mathrm{N}$-complex) (Fig. 3). ${ }^{23}$ Contrary to the $2: 1$ complex, where the VAMP2 binding site is blocked by the second copy of syntaxin-1A, this $\Delta \mathrm{N}$-complex offers a free binding site for VAMP2. It, thereby, greatly facilitates SNARE nucleation and speeds up membrane docking. The dissociation of the VAMP2 (49-96) fragment is the rate limiting step for membrane fusion and results in sigmoidal rather than exponential lipid

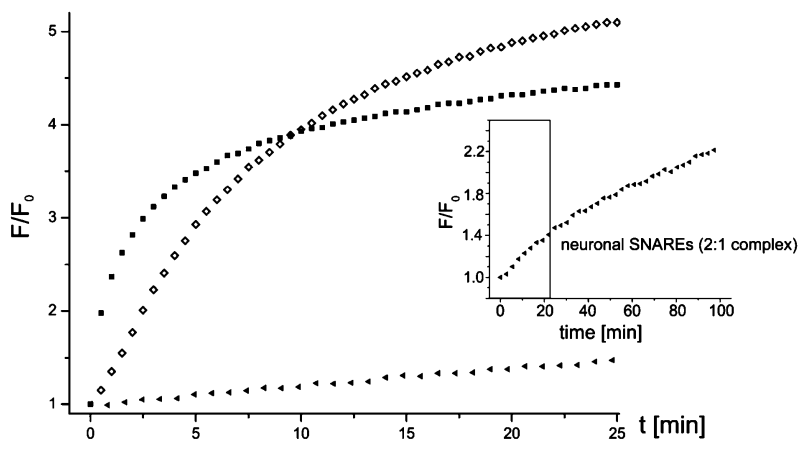

Fig. 3 Comparison of lipid mixing induced by SNARE-mimicking peptides and neuronal SNAREs: E3-VAMP2 and K3-syntaxin (ש), 2: 1 complex $(\triangleleft)$ and $\Delta \mathrm{N}$-complex $(\diamond)$ in a $1 / 1000$ peptide/lipid ratio for all populations (for definition of the $2: 1$ complex and $\Delta \mathrm{N}$-complex see text). 


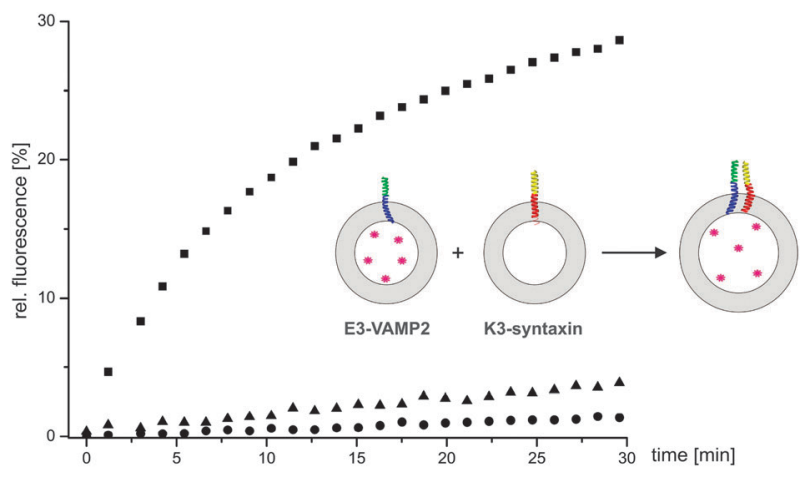

Fig. 4 Content mixing experiment with E3-VAMP liposomes filled with sulforhodamine $\mathrm{B}$ at fluorescence self-quenching concentration (20 mM). Addition of unlabelled K3-syntaxin liposomes ( $\mathbf{\square})$, liposomes with E3-VAMP $(\bullet)$, liposomes lacking a fusion peptide $(\boldsymbol{\Delta})$.

mixing kinetics (Fig. 3). ${ }^{26}$ Nevertheless, because of the dramatic enhancement of the membrane docking efficiency, the $\Delta \mathrm{N}$-complex mediates the fastest SNARE induced in vitro fusion known. ${ }^{23}$ Since our minimal fusion peptides displayed similar lipid mixing kinetics as the $\Delta \mathrm{N}$-complex, but do not require the (non-physiological) dissociation of a stabilizing peptide, we conclude that our K3-syntaxin/E3-VAMP2 mimics are well suited for further investigations of SNARE mediated membrane fusion.

The lipid mixing assay used in the experiments above does not allow distinguishing between full fusion and hemifusion where only the outer leaflet of the membrane bilayer is fused but the inner leaflet remains intact. Therefore, we carried out content mixing experiments using a self-quenching concentration of $20 \mathrm{mM}$ sulforhodamine B (SRB) encapsulated in the E3-VAMP2 liposomes. ${ }^{27-31}$ These liposomes were fused with K3-syntaxin liposomes similarly prepared but lacking the dye. Full membrane fusion, but not hemifusion, results in dilution of the encapsulated SRB. Dequenching can be detected by an increase in fluorescence. Upon addition of liposomes containing K3-syntaxin (120 $\mu \mathrm{M}$ lipids) to SRB loaded E3-VAMP2 liposomes (40 $\mu \mathrm{M}$ lipids), a $30 \%$ increase of fluorescence was observed relative to the fluorescence at the start of the experiment and the maximal fluorescence after Triton-X100 full membrane lysis (Fig. 4). As a control experiment, SRB loaded E3-VAMP2 liposomes $(40 \mu \mathrm{M})$ were mixed with liposomes $(120 \mu \mathrm{M})$ lacking the fusion peptides or with E3-VAMP2 containing liposomes $(120 \mu \mathrm{M})$. In both cases only minor changes in fluorescence were detected (Fig. 4). Therefore, it was concluded that coiled-coil formation of the membrane bound K3-syntaxin/E3-VAMP2 peptides indeed promotes full membrane fusion.

In summary, we report a novel SNARE-mimicking system in which a recognition motif containing the coiled-coil forming peptides E3/K3 was synthetically linked to the native TMDs of neuronal SNARE proteins. K3-syntaxin/E3-VAMP2 mediated membrane fusion was very efficient in both lipid mixing and content mixing assays. Therefore, these peptide constructs provide a simplified tool for studying the steps of membrane fusion of artificial membranes downstream of coiled-coil formation. Native SNAREs have a recognition motif of four domains, each about 90 amino acids in length. In contrast, the
SNARE-mimicking peptides require only an E3/K3-recognition unit two times 21 amino acids. This constitutes a nine-fold smaller size and a substantially reduced complexity. Further investigations will concentrate on modifications in the linker region investigating various sequences and lengths regarding the membrane fusion ability.

Generous support by the Deutsche Forschungsgemeinschaft (SFB 803) is gratefully acknowledged.

\section{Notes and references}

1 R. Jahn and R. H. Scheller, Nat. Rev. Mol. Cell Biol., 2006, 7, 631.

2 P. I. Hanson, R. Roth, Morisaki, R. Jahn and J. E. Heuser, Cell, 1997, 90, 523 .

3 R. C. Lin and R. H. Scheller, Neuron, 1997, 19, 1087.

4 T. H. Kloepper, C. N. Kienle and D. Fasshauer, Mol. Biol. Cell, 2007, 18, 3463.

5 T. Brunger, K. Weninger, M. Bowen and S. Chu, Annu. Rev. Biochem., 2009, 78, 903.

6 T. Weber, B. V. Zemelman, J. A. McNew, B. Westermann, M. Gmachl, F. Parlati, T. H. Söllner and J. E. Rothman, Cell, 1998, 92, 759 .

7 H. R. Marsden, I. Tomatsu and A. Kros, Chem. Soc. Rev., 2011, 40, 1572.

8 G. Stengel, R. Zahn and F. Höök, J. Am. Chem. Soc., 2007, 129, 9584 .

9 G. Stengel, L. Simonsson, R. A. Campbell and F. Höök, J. Phys. Chem. B, 2008, 112, 8264.

10 H. R. Marsden, N. A. Elbers, P. Bomans, N. A. J. M. Sommerdijk and A. Kros, Angew. Chem. Int. Ed., 2009, 48, 2330.

11 Y. M. Chan, B. van Lengerich and S. G. Boxer, Biointerphases, 2008, 3, FA17.

12 Y. H. Chan, B. van Lengerich and S. G. Boxer, Proc. Natl. Acad. Sci. U. S. A., 2009, 106, 979.

13 A. Kashiwada, M. Tsuboi and K. Matsuda, Chem. Commun., 2009, 695.

14 Y. Gong, M. Ma, Y. Luo and D. Bong, J. Am. Chem. Soc., 2008, 130, 6196.

15 Y. Gong, Y. Luo and D. Bong, J. Am. Chem. Soc., 2006, 128, 1430.

16 A. S. Lygina, K. Meyenberg, R. Jahn and U. Diederichsen, Angew. Chem. Int. Ed., 2011, 50, DOI: 10.1002/ange.201101951.

17 A. Stein, G. Weber, M. C. Wahl and R. Jahn, Nature, 2009, 460, 525 .

18 J. R. Litowski and R. S. Hodges, J. Biol. Chem., 2002, 277, 37272.

19 F. Li, F. Pincet, E. Perez, W. S. Eng, T. J. Melia, J. E. Rothman and D. Tareste, Nat. Struct. Mol. Biol., 2007, 14, 890.

20 D. K. Struck, D. Hoekstra and R. E. Pagano, Biochemistry, 1981, 20, 4093 .

21 C. G. Schuette, K. Hatsuzawa, M. Margittai, A. Stein, D. Riedel, P. Küster, M. König, C. Seidel and R. Jahn, Proc. Natl. Acad. Sci. U. S. A., 2004, 101, 2858.

22 S. Takamori, M. Holt, K. Stenius, E. A. Lemke, M. Grønborg, D. Riedel, H. Urlaub, S. Schenck, B. Brügger, P. Ringler, S. A. Müller, B. Rammner, F. Gräter, J. S. Hub, B. L. De Groot, G. Mieskes, Y. Moriyama, J. Klingauf, H. Grubmüller, J. Heuser and F. Wieland, Cell, 2006, 127, 831.

23 A. V. Pobbati, A. Stein and D. Fasshauer, Science, 2006, 313, 673.

24 F. Parlati, T. Weber, J. A. McNew, B. Westermann, T. H. Söllner and J. E. Rothman, Proc. Natl. Acad. Sci. U. S. A., 1999, 96, 12565 .

25 A. Stein, A. Radhakrishnan, D. Riedel, D. Fasshauer and R. Jahn, Nat. Struct. Mol. Biol., 2007, 14, 904.

26 A. Cypionka, A. Stein, J. M. Hernandez, H. Hippchen, R. Jahn and P. J. Walla, Proc. Natl. Acad. Sci. U. S. A., 2009, 106, 18575.

27 J. Wilschut, N. Duzgunes, R. Fraley and D. Papahadjopoulos, Biochemistry, 1980, 19,6011.

28 J. Wilschut and D. Papahadjopoulos, Nature, 1979, 281, 690.

29 J. N. Weinstein, S. Yoshikami, P. Henkart, R. Blumenthal and W. A. Hagins, Science, 1977, 195, 489.

30 B. J. Ravoo, W. D. Weringa and J. Engberts, Biophys. J., 1999, 76, 374.

31 R. El Jastimi and M. Lafleur, Biospectroscopy, 1999, 5, 133. 